\title{
Prevalence and predictors of bradyarrhythmias requiring Permanent Pacing in patients with Anderson-Fabry disease.
}

\author{
luigi tassetti ${ }^{1}$, Carlo Fumagalli ${ }^{1}$, Alessia Argirò ${ }^{1}$, Mattia Zampieri ${ }^{1}$, Martina Gori ${ }^{1}$, \\ Federica Verrillo ${ }^{1}$, Chiara Zocchi ${ }^{1}$, Francesco Cappelli ${ }^{1}$, and Iacopo Olivotto ${ }^{1}$ \\ ${ }^{1}$ University Hospital Careggi
}

November 16, 2021

\begin{abstract}
Introduction: Bradyarrhythmias are an established red flag for storage cardiac conditions including Anderson-Fabry disease (AFD). The prevalence of bradyarrhythmias requiring a pacemaker (PM) and their timing in AFD is unresolved. We evaluated prevalence and predictors of PM requirement in a large AFD cohort, investigating the occurrence of bradyarrhythmias as initial versus late manifestation. Methods: we retrospectively evaluated 82 consecutive AFD patients referred to our multidisciplinary referral centre from 1994 to 2020 with a median follow up of 6.9 years, identifying those requiring pacing. Univariable analysis was performed to identify cardiac features associated with PM implantantion. Results: Five of 82 (6\%) AFD patients required PM implantation (5/39, i.e. $13 \%$ of those with cardiac involvement), always in the context of advanced cardiomyopathy. In none, bradyarrhythmias were the presenting feature. Indications included sick sinus syndrome in 3 patients, advanced atrioventricular block in 2 patients. QRS prolongation during follow up strongly correlated with the onset of bradyarrhythmias. Conclusions: Severe bradyarrhythmias are relatively frequent in patients with AFD cardiomyopathy, but do not represent a mode of presentation, occurring late in the disease course and always in the context of advanced cardiac involvement. Monitoring QRS variations over time may help to identify patients requiring pacing.
\end{abstract}

\section{Hosted file}

manuscript Prevalence and predictors of bradyarrhythmias requiring Permanent Pacing in patients with An available at https://authorea.com/users/446352/articles/545611-prevalence-and-predictors-ofbradyarrhythmias-requiring-permanent-pacing-in-patients-with-anderson-fabry-disease 